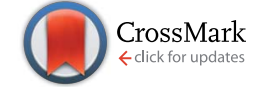

Cite this: J. Mater. Chem. A, 2016, 4, 1792

Received 28th October 2015

Accepted 4th January 2016

DOI: $10.1039 / c 5 t a 08680 a$

www.rsc.org/MaterialsA

\section{Towards sustainable solid-state supercapacitors: electroactive conducting polymers combined with biohydrogels $\uparrow$}

\author{
Maria M. Pérez-Madrigal, ${ }^{\text {abc }}$ Francesc Estrany, $^{\text {cd }}$ Elaine Armelin, ${ }^{\text {abc }}$ David Díaz Díaz ${ }^{\star a e}$ \\ and Carlos Alemán*bc
}

Solid-state organic electrochemical supercapacitors (OESCs) have been fabricated using poly(3,4ethylenedioxythiophene) (PEDOT) electrodes, a biohydrogel as electrolyte system, and polyaniline fibers as redox additive. The effectivity of sodium alginate, $\kappa$-carrageenan, chitosan and gelatin hydrogels as electrolytic media has been evaluated considering different criteria. Results indicate that $\kappa$-carrageenanbased hydrogel is the most suitable to perform as electrolyte due to the appropriate combination of properties: mechanical stability, ease of preparation, lack of water leaking, and good medium for the electrochemical response of PEDOT electrodes. Cyclic voltammetry and galvanostatic charge-discharge assays indicate that OESCs based on PEDOT electrodes and $\kappa$-carrageenan hydrogel as electrolyte exhibits a good supercapacitor response in terms of specific capacitance, cycling stability, small leakage current and low self-discharging tendency. On the basis of these good properties, four OESC devices were assembled in series and used to power a red LED, confirming that, in addition to advantageous characteristics (e.g. elimination of liquid leaking and enhancement of the device compactness), the designed biohydrogel-containing OESC exhibits potential for practical applications. On the other hand, preliminary assays have been performed loading the $\kappa$-carrageenan hydrogel with polyaniline nanofibers, which act as a redox additive. OESC devices prepared using such loaded biohydrogel have been found to be very promising and, therefore, future work is oriented towards the improvement of their design.

\section{Introduction}

Global concerns on green-chemistry and sustainable development have oriented research and technological developments towards more eco-friendly approaches. This is particularly important for energy-related issues due to both the depletion of fossil fuels and the negative environmental effects that they generate. Within this context, efforts are being made to (i) shift to renewable energy sources; and (ii) design high-performance energy storage devices (i.e. batteries and capacitors) using ecofriendly materials. Batteries display low-power densities

${ }^{a}$ Institut für Organische Chemie, Fakultät für Chemie und Pharmazie, Universität Regensburg, Universitätsstr. 31, 93053 Regensburg, Germany. E-mail: david.diaz@ chemie.uni-Regensburg.de

${ }^{b}$ Departament d'Enginyeria Química, ETSEIB, Universitat Politècnica de Catalunya, Avda. Diagonal 647, Barcelona E-08028, Spain.E-mail: carlos.aleman@upc.edu

${ }^{c}$ Center for Research in Nano-Engineering, Universitat Politècnica de Catalunya, Campus Sud, Edifici C', C/Pasqual $i$ Vila $s / n$, Barcelona E-08028, Spain

${ }^{d}$ Departament d'Enginyeria Química, Escola Universitària d'Enginyeria Tècnica Industrial de Barcelona, Universitat Politècnica de Catalunya, Comte d'Urgell 187, 08036 Barcelona, Spain

${ }^{e} I Q A C-C S I C$, Jordi Girona 18-26, E-08034 Barcelona, Catalonia, Spain

$\dagger$ Electronic supplementary information (ESI) available. See DOI: 10.1039/c5ta08680a although they have high-energy densities, whereas the opposite is true for conventional capacitors. ${ }^{\mathbf{1}}$ In between, electrochemical capacitors, also called supercapacitors (ESCs), have recently been designed with advanced functionalities via environmentally-friendly processes. These systems exhibit high power and energy densities without compromising their cycling stability or other features. ${ }^{2-4}$ Furthermore, the development of nanostructured electrode materials and the better understanding of the energy-storage mechanisms have contributed to improve the performance of ESCs and to bridge the gap between electrochemical capacitors and batteries in terms of energy density. ${ }^{5-10}$

Depending on the energy-storage mechanism, electrode materials are divided in two different main groups. In the first one the capacitive behaviour solely relies on the electrostatic interactions (adsorption) between electrolytic ions and the electrode surface (i.e. electrical double-layer capacitance), while faradaic reactions contribute to the overall capacitance (i.e. pseudocapacitance) in the second group. ${ }^{11}$ Electroactive conducting polymers (ECPs) belong to the latter group and render promising electrodes in organic electrochemical supercapacitors (OESCs) due to their cost-effectiveness, lightweight and flexibility. ${ }^{\mathbf{1 2}}$ In fact, ECPs display pseudocapacitance behavior as a result of: (i) the redox mechanism (i.e. charge is 
stored by electron transfer to the polymer with ion pairing to stabilize the charge); and (ii) the double-layer behavior of doped ECPs, which allows resonance throughout the conjugated structure of the polymeric backbone. In a recent work, Mike and Lutkenhaus ${ }^{\mathbf{1 3}}$ extensively reviewed the advances of ECPs in the energy storage field. Among ECPs, poly(3,4-ethylenedioxythiophene) (PEDOT) has been widely used for the fabrication of OESCs because of its outstanding capacitive performance, good electrical conductivity, high doping rate, stable charge-discharge response, easy of synthesis and advantages in cost. ${ }^{\text {14-18 }}$

On the other hand, ECP-based hydrogels are receiving increasing attention, ${ }^{\mathbf{1 8 , 1 9}}$ specially for their application as electrodes in OESCs devices..$^{20-23}$ For example, multifunctional polyaniline (PAni) hydrogels, with high surface area and threedimensional porous nanostructure, displayed excellent electronic conductivity and electrochemical properties. ${ }^{22}$ As supercapacitors, such PAni hydrogels exhibited high specific capacitance and cycling stability. Similarly, the specific capacitance displayed by nanostructured conductive polypyrrole (PPy) hydrogels supported their application as supercapacitor electrodes. ${ }^{23}$

Eco-friendly innovative strategies have been undertaken to improve the performance of energy storage devices considering different strategies, ${ }^{24-30}$ as for example the design of both electrode materials and redox electrolytes ${ }^{24}$ using natural resources for electrode materials (e.g. PAni in sodium alginate $)^{25}$ and aqueous-based solutions as electrolytic system (e.g. $\mathrm{KOH}, \mathrm{H}_{2} \mathrm{SO}_{4}$ and $\left.\mathrm{Na}_{2} \mathrm{SO}_{4}\right) .{ }^{26}$ Regarding the electrolyte system, solid-state supercapacitors, which are formed not only by solid electrodes but also by a solid electrolyte system (SES), have attracted increasing attention because of their advantageous characteristics: high specific/volumetric energy density, flexibility, light-weight and easy of handling in comparison to conventional capacitors. $^{31,32}$ Furthermore, SES reduce or eliminate leakage of any liquid component and increase the device compactness.

Polymeric materials used as SESs can be classified in three groups: (1) gel-based materials (e.g. polyacrylamide or agar); (2) polymers with dissolved salts (e.g. lithium perchlorate in polyethylene oxide); and (3) polyelectrolytes. Interestingly, recent studies focus on eco-friendly electrolytes for high-performance solid-state supercapacitors. For example, Buchtová et $a l^{33}$ prepared an ionogel based on a chemically cross-linked polysaccharide matrix and a hydrophobic ionic liquid as a greener alternative to petrochemical-based polymeric SESs. Choudhury et $a l .{ }^{34}$ reviewed that gel-polymer electrolytes frequently require using organic solvents during the gelation process, which is environmentally malignant. Hence, replacement of organic solvents with water in these electrolytic systems is highly desirable in terms of sustainability, also minimizing the device cost substantially. However, water-containing gel-polymer electrolytes - hydrogel-polymer electrolytes - limit the operating potential-window (i.e. $\sim 1.23 \mathrm{~V}$ only). ${ }^{34}$ Within this context, the performance of some ESCs has been improved using biohydrogels (e.g. sodium alginate, hydroxycellulose, gelatin and chitosan). ${ }^{34-37}$ For example, the electrochemical performance of the $\mathrm{Si} / \mathrm{C}$ anode of $\mathrm{Li}$-ion batteries was enhanced by applying a cross-linked alginate hydrogel binder. ${ }^{35}$

This work develops greener and more sustained energy storage devices by replacing conventional liquid electrolytic systems with biohydrogels in OESCs. If properly designed, biohydrogels are safe (i.e. non-toxic, non-flammable, and nonleakage), cheap and easy to prepare; they exhibit high-ionic conductivities and can be obtained from renewable sources. In spite of the advantageous outcome of biohydrogel-based systems as sustainable energy storage devices, caution is required since the electrolyte system plays a crucial role in determining the capacitor response. Thus, if not appropriately conceived, the ion-diffusion rate in hydrogels may be poorer than that shown by liquid electrolytes, and thus limiting the final application of the energy storage device. In this work, $\mathrm{Na}$ alginate, $\kappa$-carrageenan, chitosan and gelatin hydrogels (Scheme S1 $\dagger$ ) have been combined with ECPs to fabricate OESC devices. More specifically, after a two-step selection process, the most appropriate biohydrogel constituted the electrolyte in a device containing PEDOT electrodes and PAni nanofibers (PAni-nfs) as redox additive.

\section{Methods}

\section{Materials}

For the synthesis of PEDOT electrodes, 3,4-ethylenedioxythiophene (EDOT, 97\%) was purchased from SigmaAldrich (483028); acetonitrile (Reag. Ph. Eur. for analysis, ACS) was purchased from Panreac (Spain); $\mathrm{LiClO}_{4}$ from SigmaAldrich (ACS reagent, $\geq 95.0 \%$ ) was stored in an oven at $70{ }^{\circ} \mathrm{C}$ before use. For the synthesis of PAni nanofibers, aniline (ACS reagent $\geq 99.5 \%$ ) and ammonium peroxodisulfate (ACS reagent $\geq 98.0 \%$ ) were purchased from Sigma-Aldrich.

The biopolymers used were all purchased from SigmaAldrich. Specifically, alginic acid sodium salt from brown algaelow viscosity (A2158, 100-300 cP, 2\% (25 $\left.{ }^{\circ} \mathrm{C}\right)$ ), $\kappa$-carrageenan sulfated plant polysaccharide $\left(22048,5-25 \mathrm{mPa} \mathrm{s}, 0.3 \%\right.$ in $\mathrm{H}_{2} \mathrm{O}$ $\left(25{ }^{\circ} \mathrm{C}\right)$ ), gelatin from porcine skin (gel strength 300 , type A G2500), and chitosan medium molecular weight (448 877, 200$800 \mathrm{cP}, 1 \mathrm{wt} \%$ in $1 \%$ acetic acid, $25{ }^{\circ} \mathrm{C}$ ).

Other reagents used during the preparation of biohydrogelbased electrolytes included: sodium chloride coarse salt QP (Panreac Química S.L.U., Spain), potassium chloride GR (Merck), maleic anhydride (Fluka Analytical, puriss., $\geq 99.0 \%$ $\mathrm{NT}$ ), formaldehyde solution (ACS reagent, 37 wt $\%$ in $\mathrm{H}_{2} \mathrm{O}$, contains 10-15\% methanol as stabilizer, Sigma-Aldrich), acetic acid min. 99.5\% (POCH), ethanol 96\% v/v (USP, BP, Ph.Eur. pure-pharma grade) and sodium hydroxide (pellets) both from Panreac Química S.L.U., Spain.

\section{Materials preparation}

PEDOT electrodes were prepared by chronoamperometry (CA) under a constant potential of $1.40 \mathrm{~V}$ and using $\mathrm{LiClO}_{4}$ as supporting electrolyte. Details about the preparation procedure are provided in the ESI. $\uparrow$ The polymerization charge was adjusted to 
$2.67 \mathrm{C}\left(445 \mathrm{mC} \mathrm{cm}^{-2}\right)$, which corresponded to a mass of polymerized PEDOT of $1.69 \pm 0.23 \mathrm{mg}$.

A brief description of Na-alginate, $\kappa$-carrageenan, gelatin and chitosan as well as of the procedures used for the preparation of their hydrogels is provided in the ESI. $\dagger$

\section{Preparation of OESC energy storage devices}

As a general procedure, OESC devices with biohydrogel-based electrolytes were prepared 24 hours before testing, and the electrolyte gelation proceeded with the electrodes placed in situ to achieve both a better contact between PEDOT and the hydrogel at the electrode/electrolyte interface, and to preserve the integrity of the ECP film (i.e. in some cases, as for example $\kappa-$ carrageenan and gelatin, insertion of the PEDOT electrode after the gelation step would damage the ECP film due to the rigidity of the hydrogel). Moreover, to ensure a correct functioning of the biohydrogel as electrolyte, $0.1 \mathrm{M} \mathrm{NaCl}$ was mixed in the biopolymer solution prior to gelation.

PEDOT electrodes were correctly fixed inside the OESC container (i.e. glass vial or plastic cup for devices with two- or three-electrode configuration, respectively, Scheme $\mathrm{S} 2 \dagger$ ), leaving $1 \mathrm{~cm}$ of separation between them. Teflon ${ }^{\circledR}$ films were used to limit the area of PEDOT $\left(6 \mathrm{~cm}^{2}\right)$, and thus prevent the exposure of steel and its contacting with the hydrogel. Once the biopolymer solution was prepared (ESI $\dagger$ ), the container was filled with approximately $20-25 \mathrm{~mL}$ to fully cover the electrodes. Except for chitosan, all OESC devices were prepared following such steps, albeit slight differences arose during the gelation step according to each procedure, namely regarding the gelation temperature (ESI $\dagger$ ). Due to the poor mechanical properties of chitosan hydrogel (see next section), for chitosan-containing devices, PEDOT electrodes and the reference electrode (when required) were inserted with extremely caution into the biohydrogel electrolyte after gelation. Finally, OESC devices were maintained at low temperature $\left(4-6{ }^{\circ} \mathrm{C}\right.$, fridge) before their electrochemical characterization.

In case of the three-electrode configuration, the reference electrode was not placed in the container before the gelation step, but a glass tube with open ends was used instead (Scheme $\mathrm{S} 2 \mathrm{~b} \dagger$ ). 1-2 $\mathrm{mL}$ of phosphate buffered solution (PBS, $\mathrm{pH}$ 7.4) were introduced in the glass tube, and let to diffuse through the electrolyte hydrogel overnight to provide a good contact between the biohydrogel-based electrolyte and the reference electrode when introduced in the glass tube prior the electrochemical assays.

Hereafter, the OESC devices with biohydrogel-based electrolyte using Na-alginate, $\kappa$-carrageenan, chitosan and gelatin will be referred as OESC-NaAlg, OESC- $\kappa \mathrm{C}$, OESC-Chi and OESCGel, respectively.

\section{Characterization}

The ESI $\dagger$ provides details of the equipment and conditions used for characterization by optical microscopy, UV-vis spectroscopy, scanning electron microscopy (SEM) and atomic force microscopy (AFM), as well as for electrical conductivity measurements.

\section{Electrochemical characterization}

Electrochemical cells in three- and two-electrode configurations were assembled for electrochemical characterization: the former was suitable for the fast characterization of the electrode material (i.e. characterization of the PEDOT working electrode), whereas the latter was used to evaluate the supercapacitor performance of OESC devices under specific working conditions (i.e. the response of the whole cell).

Accordingly, the electrochemical response of PEDOT in different biohydrogel electrolytic media was first studied using a three-electrode cell configuration. The counter electrode (CE) was made of bare stainless steel, while an $\mathrm{Ag} \mid \mathrm{AgCl}$ electrode containing saturated $\mathrm{KCl}$ aqueous solution $\left(E^{0}=0.222 \mathrm{~V} v\right.$ s. standard hydrogen electrode at $25{ }^{\circ} \mathrm{C}$ ) was used as reference electrode (RE). The electrochemical activity and stability (electroactivity and electrostability, respectively) of PEDOT in the different electrolytic media were determined by cyclic voltammetry (CV) after applying 50 consecutive oxidation-reduction cycles. The initial and final potential was $-0.2 \mathrm{~V}$, whereas the reversal potential was $1.0 \mathrm{~V}$. All measurements were performed at room temperature using a scan rate of $50 \mathrm{mV} \mathrm{s}^{-1}$. The electrostability was quantified as the loss of electroactivity (LEA; in\%):

$$
\mathrm{LEA}=\frac{\Delta Q}{Q_{\mathrm{II}}} \times 100=\frac{Q_{2}-Q_{\#}}{Q_{2}}
$$

where $\Delta Q$ is the difference between the oxidation charge (in $\mathrm{C}$ ) of the second $\left(Q_{2}\right)$ and the evaluated oxidation-reduction cycle $\left(Q_{\#}\right)$. Data were obtained as the average of three samples for each system.

Secondly, the performance of OESC devices $(\mathrm{WE}=\mathrm{CE}=$ PEDOT) using different biohydrogel electrolytic media was evaluated in a two-electrode configuration. Galvanostatic chargedischarge (GCD) cycles were run between 0.0 and $0.8 \mathrm{~V}$. Different current densities (i.e. charge and discharge rates, which are specified in units of current per electrode mass) were considered: $0.276,0.692,1.382,2.764$ or $5.529 \mathrm{~A} \mathrm{~g}^{-1}$ that corresponded to 0.467, 1.168, 2.336, 4.672 and $9.344 \mathrm{~mA}$, respectively. All electrochemical experiments were run in triplicate and results presented in this work correspond to the averaged data.

The specific capacitance ( $\mathrm{SC}, \mathrm{F} \mathrm{g}^{-1}$ ), which is the capacitance per unit of mass for one electrode, was derived from the GCD assays and calculated by applying the following expression:

$$
\mathrm{SC}=4 \frac{C}{m}
$$

where $C$ (in F) is the measured capacitance for the two-electrode cell and $m$ the total mass of the active material in both electrodes (i.e. $3.38 \times 10^{-3} \mathrm{~g}$ ). The mass of PEDOT deposited during polymerization was determined as the weight difference between coated and uncoated steel sheets (ESI $\dagger$ ). The multiplier 4 adjusts the capacitance of the cell and the combined mass of the two electrodes to the capacitance and mass of a single electrode. ${ }^{38}$ The cell capacitance was obtained from the GCD curves using the following equation:

$$
C=\frac{I}{(\mathrm{~d} V / \mathrm{d} t)}
$$


where $I$ is the discharging current applied to the device. As the pseudocapacitance varies with the voltage, $\mathrm{d} V / \mathrm{d} t$ was calculated as $\left(V_{\max }-1 / 2 V_{\max }\right) /\left(t_{2}-t_{1}\right)$, where $V_{\max }$ corresponds to the highest voltage in the GCD curve after the voltage drop ( $\left.V_{\text {drop }}\right)$ at the beginning of the discharging process.

Although GCD evaluation is the procedure most commonly used to determine the SC values of a pseudocapacitor, $\mathrm{CV}$ can also provide this information. Cyclic voltammograms were recorded from 0.0 to $0.8 \mathrm{~V}$ after five consecutive redox cycles at scan rates of $10,25,50,75,100,150$ and $200 \mathrm{mV} \mathrm{s}^{-1}$. Again, by applying eqn (2) and (3) the SC of the OESC device can be determined. In this case, $I$ is considered as the average current during the discharge step (from $0.8 \mathrm{~V}$ to $0 \mathrm{~V}$ ); and $\mathrm{d} V / \mathrm{d} t$ is the scan rate used.

The coulombic efficiency $(\eta, \%)$ of OESC devices was evaluated as the ratio between the discharging and charging times $\left(t_{\mathrm{d}}\right.$ and $t_{\mathrm{c}}$, respectively) for the electrochemical window between 0.0 $\mathrm{V}$ and $0.8 \mathrm{~V}$ :

$$
\eta=\frac{t_{\mathrm{d}}}{t_{\mathrm{c}}}
$$

The cycling stability of the as-prepared OESC devices was studied by applying 500 and 3000 charge-discharge cycles at a current density of $1.382 \mathrm{~A} \mathrm{~g}^{-1}$ in the two-electrode configuration from 0.0 to $0.8 \mathrm{~V}$, which corresponds to $t_{\mathrm{c}}$ and $t_{\mathrm{d}}$ of approximately 20-23 seconds. It is recommended that the current should be adjusted to provide charge and discharge times in the range between 5 and 60 seconds. ${ }^{38}$ The timedependence evolution of the OESC electrochemical characteristics was determined after 1 week by repeating the GCD test (500 cycles).

Finally, the maximum energy density $\left(E_{\max }, \mathrm{W} \mathrm{h} \mathrm{kg}^{-1}\right)$, which is the amount of energy stored per unit of mass, and the power density $\left(P_{\max }, \mathrm{W} \mathrm{kg}^{-1}\right)$, which describes the speed at which the energy stored can be delivered, can be derived from the GCD curves by applying the following equations: ${ }^{39}$

$$
\begin{gathered}
E_{\max }=\frac{1}{2} \times V_{\max }^{2} \times \mathrm{SC} \\
P_{\max }=\frac{1}{4 m} \frac{V_{\text {max }}^{2}}{R_{\mathrm{i}}} \\
R_{\mathrm{i}}=\frac{V_{\text {drop }}}{I_{\text {discharge }}}
\end{gathered}
$$

where $V_{\max }$ corresponds to the highest voltage in the GCD curve after the voltage drop at the beginning of the discharging process, $m$ is the total mass of the active material in both electrodes (i.e. $3.38 \mathrm{mg}$ ) and $R_{\mathrm{i}}$ is the internal resistance.

Moreover, the evaluation of the leakage current and self-discharging curves of selected OESC devices has been carried out. In the first case, after charging the device to $0.8 \mathrm{~V}$ at $2.334 \mathrm{~mA}$ (i.e. $1.381 \mathrm{~A} \mathrm{~g}^{-1}$ ), it was kept at $0.8 \mathrm{~V}$ for $5 \mathrm{~min}$ while recording the current data through the OESC (i.e. leakage current). In the second case, the OESC was charged to $0.8 \mathrm{~V}$ at $2.334 \mathrm{~mA}$ and kept at $1 \times 10^{-11} \mathrm{~mA}$ for $10 \mathrm{~min}$ (self-discharging). After that time, the device was discharged to $0.2 \mathrm{~V}$ at $-1.0 \mathrm{~mA}$.

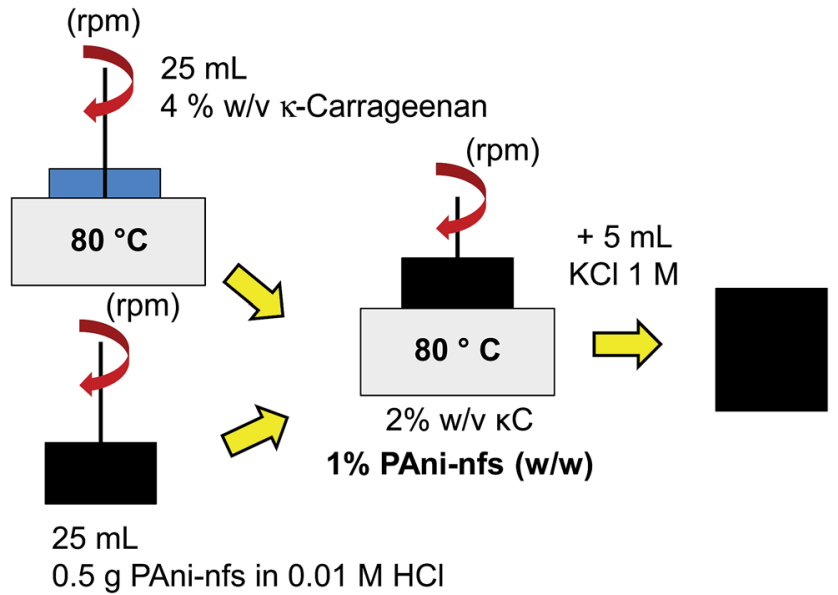

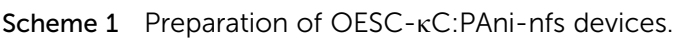

Preparation and characterization of OESC energy storage devices with PAni nanofibers-containing biohydrogels

PAni nanofibers (PAni-nfs), which were prepared using the procedure described in the ESI, $\dagger$ were introduced at bulk $\kappa$ carrageenan hydrogel using the procedure described in Scheme 1. $\kappa$-Carrageenan was dissolved in water $(25 \mathrm{~mL})$ at $4 \% \mathrm{w} / \mathrm{v}$ at $c a$. $75-80{ }^{\circ} \mathrm{C}$. On the other hand, a solution of $0.5 \mathrm{~g}$ PAni-nfs was prepared in $25 \mathrm{~mL}$ of $0.01 \mathrm{M} \mathrm{HCl}$. After dispersing PAni-nfs by ultrasonication (10 $\mathrm{min}$ ), both solutions were mixed and stirred for $10 \mathrm{~min}$. Then, the corresponding volume of $1 \mathrm{M} \mathrm{KCl}(10 \% \mathrm{v} /$ v) was added, and the solution stirred vigorously again. The gel was formed at room temperature for several hours. Finally, OESC energy store devices were prepared as described previously, even though in this case the hydrogel-based electrolyte was loaded with PAni-nfs $(1 \% \mathrm{w} / \mathrm{w})$.

Initially, the electrochemical response of PEDOT in biohydrogels loaded with PAni-nfs was preliminary examined in a three-electrode configuration using bare stainless steel as CE and a $\mathrm{Ag} \mid \mathrm{AgCl}$ electrode containing saturated $\mathrm{KCl}$ aqueous solution $\left(E^{0}=0.222 \mathrm{~V} v\right.$ s. standard hydrogen electrode at $\left.25^{\circ} \mathrm{C}\right)$ as RE. The electroactivity and electrostability (eqn (1)) of PEDOT were determined by $\mathrm{CV}$, applying 50 consecutive oxidationreduction cycles. The initial and final potential was $-0.4 \mathrm{~V}$, while the reversal potential was $1.0 \mathrm{~V}$. All measurements were performed at room temperature using a scan rate of $50 \mathrm{mV} \mathrm{s}^{-1}$. After these electrochemical measurements, the applicability of the PAni-nfs-containing OESC was examined.

\section{Results and discussion}

\section{Preparation of PEDOT electrodes}

PEDOT electrodes were obtained by chronoamperometry (CA). In this electrochemical technique the potential of the working electrode is stepped and the current resulting from faradaic processes at the electrode (caused by the potential step) is monitored as a function of time. For this study the employed potential was $1.40 \mathrm{~V}$ and a polymerization charge of $2.67 \mathrm{C}$ was imposed (details provided in the ESI $\dagger$ ). Under these 
electrochemical conditions, as prepared PEDOT films exhibited a thickness of $4.25 \pm 0.36 \mu \mathrm{m}$, an electrical conductivity of 0.52 $\pm 0.16 \mathrm{~S} \mathrm{~cm}^{-1}$, and a mass of $1.69 \pm 0.23 \mathrm{mg}$. The morphology and surface topography of thin film electrodes (i.e. those with a thickness below $10 \mu \mathrm{m}$ ) affect the SC and ion diffusion characteristics of nanostructured capacitors. ${ }^{17,38,40}$ For example, the SC of PEDOT electrochemically polymerized thin films is $c a .100$ $\mathrm{F} \mathrm{g} \mathrm{g}^{-1},{ }^{41}$ decreasing to $<20 \mathrm{~F} \mathrm{~g}^{-1},<60 \mathrm{~F} \mathrm{~g}^{-1}$ or $<100 \mathrm{~F} \mathrm{~g}^{-1}$ when designed as tracked blocks, ${ }^{42}$ nanoparticles ${ }^{43}$ or micro/nanorods, ${ }^{44}$ respectively. Within this context, it should be emphasized that the SC of ECP-based electrodes can be significantly enhanced with respect to these values by changing the working electrode (e.g. replacing the steel electrode using in this work by nickel, copper, gold or platinum), optimizing the oxidizing agent $\left(\mathrm{LiClO}_{4}\right.$ in this work), adding metallic or ceramic nanoparticles, carbon nanotubes or graphene, etc. ${ }^{45}$ However, this work is focused on the replacement of the liquid electrolytic medium by a selected biohydrogel and, therefore, a simple PEDOT electrode has been used during the whole work.

Fig. S1 and S2† display details on the morphological and topographical characterization of prepared PEDOT electrodes. In relation to the morphology, PEDOT films present a spongelike surface with pores that facilitate the access and escape of electrolyte ions during electrochemical redox processes (Fig. S1†). Hence, this morphology results in thin-films with a continuous open structure and void spaces that, if properly infiltrated by the biohydrogel-based electrolyte during the gelation step, would enhance the pseudocapacitance of the OESC device.

AFM images are in good agreement with SEM observations. Specifically, cross-section profiles evidence that PEDOT aggregates of varying size and height (between 1 and $3 \mu \mathrm{m}$ ) are distributed throughout the surface. In consequence, the root mean square roughness $\left(R_{\mathrm{q}}\right)$ of the surface varies depending on the analysed area: from $487 \pm 82 \mathrm{~nm}$ for areas $\left(5 \times 5 \mu \mathrm{m}^{2}\right)$ with a relatively homogenous topography to $635 \pm 79 \mathrm{~nm}$ for those areas $\left(20 \times 20 \mu^{2}\right)$ in which bigger clusters are identified (Fig. S2†).

It is worth noting that the pseudocapacitance response of ECPs electrodes with porous morphology and large specific surface is favoured since these structures promote fast ion and electron transportation, which in turn allow for higher charge storage and release by faradaic reactions. Although both SEM and AFM characterization techniques have provided reliable information of the morphology and surface topography of PEDOT electrodes, Brunauer-Emmett-Teller (BET) analyses would allow to report specific values for the surface area and porosity. Unfortunately, in this case the BET analysis was not a feasible technique because of the limitations in the mass of PEDOT produced by the electrochemical synthetic route. Indeed, it is worth noting that the amount of works specifying quantitatively the surface area and porosity is relatively scarce $^{46-50}$ probably due to the limitations of the BET test. Thus, the morphology of PEDOT electrodes for energy storage applications is frequently described qualitatively by examining SEM micrographs, especially when the polymer is generated by anodic polymerization..$^{51,52}$
In a very recent study Zhang and Suslick ${ }^{46}$ reported the preparation of PEDOT microspheres by polymerizing nebulized microdroplets of solutions containing EDOT monomers and oxidants, using an ultrasonic spray polymerization (USPo) technique. ${ }^{46}$ The BET surface area of the porous microspheres obtained in presence of $\mathrm{FeCl}_{3}$ was $49 \mathrm{~m}^{2} \mathrm{~g}^{-1}$, which was comparable to the surface area of nanoporous PEDOT monoliths $\left(26 \mathrm{~m}^{2} \mathrm{~g}^{-1}\right)$ reported in a previous work. ${ }^{47}$ It should be emphasized that Zhang and Suslick used such spraying technology to coat flat surfaces of silicon wafer, the morphology of the resulting PEDOT coating being similar to the one displayed by our PEDOT electrodes in Fig. S1. $\uparrow$ Because of that, we consider that a BET surface area value reported by Zhang and Suslick ${ }^{46}$ (ca. 30-50 $\left.\mathrm{m}^{2} \mathrm{~g}^{-1}\right)$ may be extrapolated to our system.

On the other hand, Han et al. ${ }^{48}$ prepared composites based on graphite oxide (GO) and PEDOT composites as supercapacitors. The presence of GO was expected to reinforce the stability of PEDOT and maximize its pseudocapacitance. In this case, PEDOT was obtained using in situ chemical oxidative polymerization. The BET specific surface area of the resulting GO/PEDOT composites was $19 \mathrm{~m}^{2} \mathrm{~g}^{-1} .^{48}$ Mukkabla et al. ${ }^{49}$ compared the redox pseudocapacitive behavior of $\mathrm{NiS}_{2}$ microspheres embedded in a PEDOT layer with that of pristine PEDOT. The electrodes were prepared electrochemically under potentiostatic conditions using an ionic liquid. SEM micrographs evidenced the presence of irregular particles of a few microns in size that were distributed randomly throughout the surface of pristine PEDOT films. Because of that, the BET surface area of pristine PEDOT films was found to be very low $\left(2 \mathrm{~m}^{2} \mathrm{~g}^{-1}\right) .{ }^{49}$ Therefore, even though the electrochemical preparation of PEDOT electrodes is similar to ours, the morphology of the resulting films is not comparable and, therefore, our PEDOT electrodes are expected to show greater surface area values. However, the scarce number of publications reporting this parameter obliges to take it under consideration. Mukkabla et al. ${ }^{50}$ have also reported the design of $\mathrm{Bi}_{2} \mathrm{~S}_{3} / \mathrm{GO}$ composites enwrapped by a PEDOT coating as the anode in Li-ion batteries, $\mathrm{Bi}_{2} \mathrm{~S}_{3} / \mathrm{GO} / \mathrm{PEDOT}$ composite films being prepared by chemical polymerization. The BET specific surface areas of pristine $\mathrm{Bi}_{2} \mathrm{~S}_{3}, \mathrm{Bi}_{2} \mathrm{~S}_{3} / \mathrm{GO}$ and $\mathrm{Bi}_{2} \mathrm{~S}_{3} / \mathrm{GO} /$ PEDOT were 12,51 and $46 \mathrm{~m}^{2} \mathrm{~g}^{-1}$, respectively, while the pore volumes were $0.008,0.034$, and $0.035 \mathrm{~cm}^{3} \mathrm{~g}^{-1}$, respectively.

Finally, although the study of Chao et al. ${ }^{53}$ does not involve the use of PEDOT, it deserves special consideration because of the morphology of the studied system. More specifically, porous $\mathrm{SnS}$ and $\mathrm{SnS}_{2}$ architectures were produced with a morphology that closely resembles the one displayed by the PEDOT electrodes designed in this work: a porous flower-like microstructure. BET analyses revealed that the surface areas of porous SnS and $\mathrm{SnS}_{2}$ structures are $66 \mathrm{~m}^{2} \mathrm{~g}^{-1}$ and $33 \mathrm{~m}^{2} \mathrm{~g}^{-1}$, respectively. ${ }^{53}$

In summary, although the surface area of the PEDOT electrodes used in this work has not been determined due to the operational restrictions of the BET test, the revised literature described above allows us to suggest that this value would be found between 30 and $50 \mathrm{~m}^{2} \mathrm{~g}^{-1}$. 
(a)

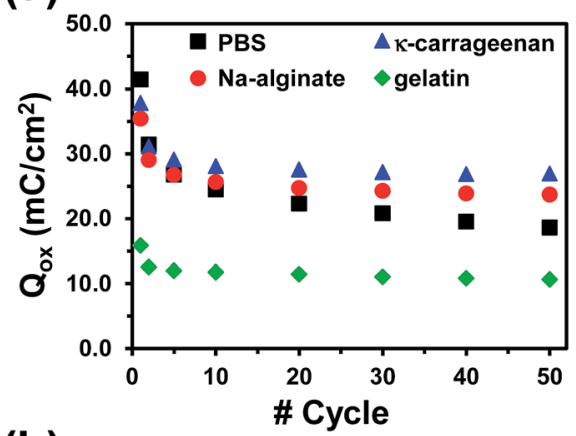

(b)
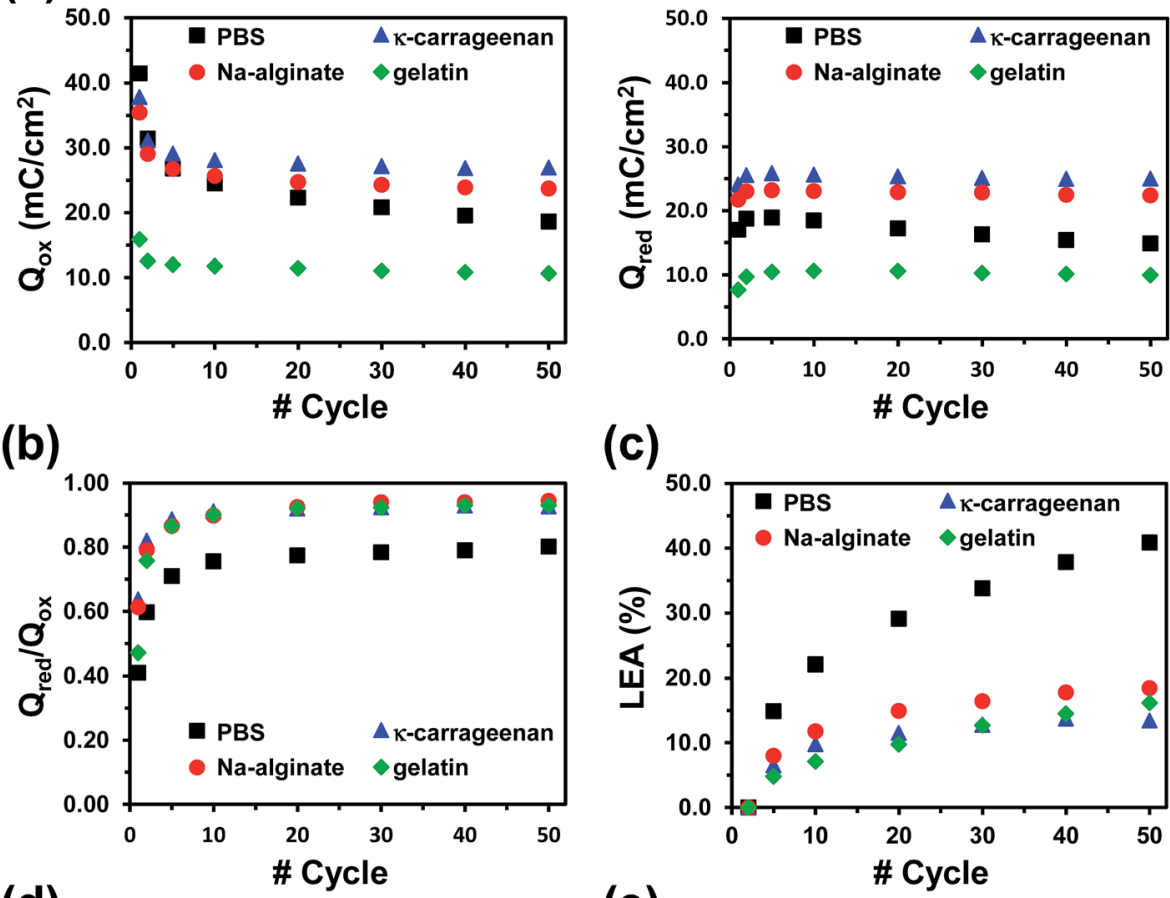

(d)

(c)
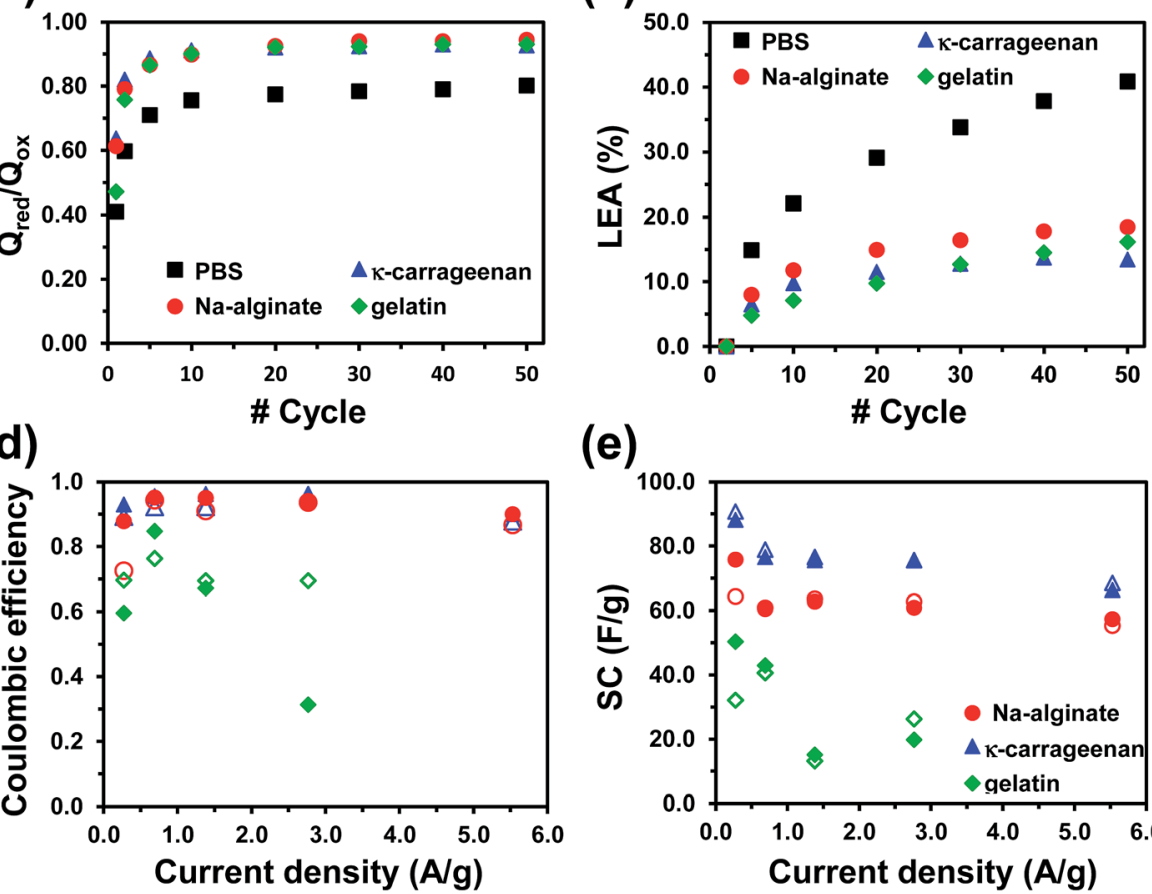

(e)

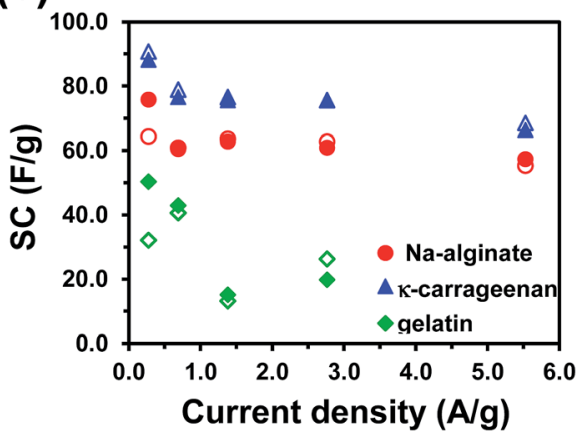

Fig. 1 Cyclic voltammetry using a three-electrode configuration (Fig. S5†) have been used to determine the following electrochemical properties for PEDOT electrodes using different electrolyte systems: (a) variation of the stored charge, where $Q_{\mathrm{ox}}$ (left) and $Q_{\text {red }}$ (right) refer to oxidation and reduction charges; (b) electrochemical activity, which is defined by the $Q_{\text {red }} / Q_{o x}$ ratio; and (c) loss of electrochemical activity (LEA) against the number of consecutive redox cycles. Data are the average of three samples for each system. Galvanostatic charge-discharge assays using a two-electrode configuration (Fig. S6†) have been employed to evaluate the variation of (d) the coulombic efficiency and (e) the specific capacitance (SC) against the applied current density for OESC devices with different biohydrogel-based electrolytes.

Preparation and selection of biohydrogels as electrolyte system

Fig. S3a and S3b $\uparrow$ display images of the prepared hydrogels (bulk) and of the OESC-biohydrogel devices in a two-electrode configuration, respectively. Hydrogel-based electrolytes derived from Na-alginate and chitosan are opaque and exhibit poor mechanical integrity (i.e. they disintegrate when submitted to stress) in comparison to those obtained using $\kappa$-carrageenan and gelatin. In fact, the extremely poor mechanical integrity of chitosan required careful handling, limiting drastically its applicability as biohydrogel-based electrolyte. In addition, water release was observed for Na-alginate and chitosan hydrogels, which also represented an important drawback. In contrast, $\kappa-$ carrageenan and gelatin hydrogels are transparent and more robust, facilitating the assembly of PEDOT electrodes into the electrolyte. According to these results, chitosan hydrogels were discarded as electrolytic system. At this point, it should be clarified that the application of the biohydrogels as electrolytic media does not require careful evaluation of their mechanical properties but only visual inspection to ensure that these materials are robust enough to keep the electrodes at their positions.

The morphology of $\kappa$-carrageenan, Na-alginate and gelatin hydrogels was characterized by SEM (Fig. S4 $\dagger$ ). Solid-state electrolytes are expected to present an open porous structure able to exploit at maximum the pseudocapacitance of PEDOT electrodes, favouring ion movement and transfer. Both $\kappa-$ carrageenan and Na-alginate hydrogels accomplish this requirement, while gelatin exhibits a compact morphology in which the interconnected network of pores is absent. 

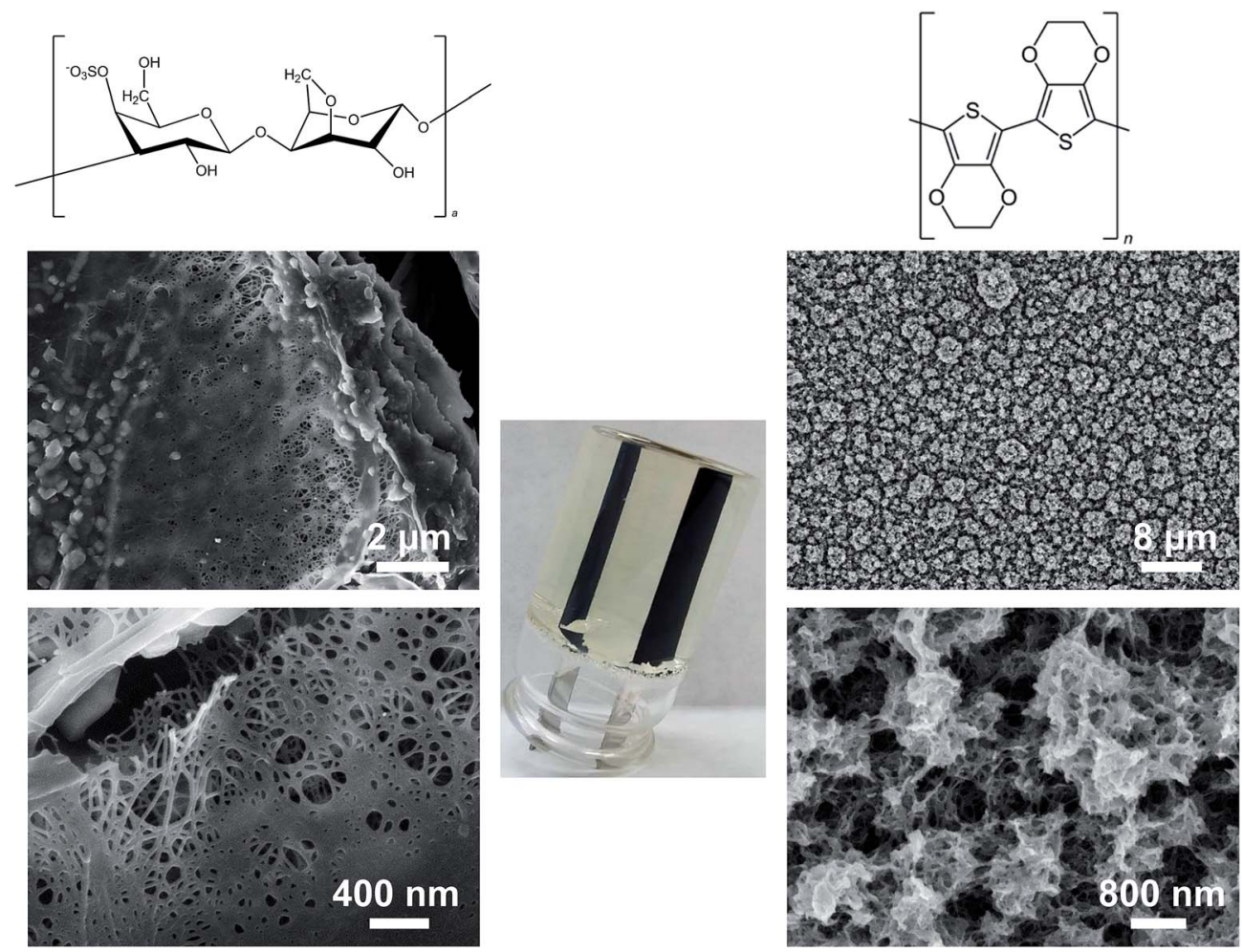

к-Carrageenan

\section{PEDOT}

Fig. 2 Chemical structure and morphology (SEM micrographs) of OESC- $\kappa C$ (middle) elements: $\kappa$-carrageenan hydrogel as electrolyte (left column) and PEDOT electrodes (right column).

\section{Selection of the sustainable OESC using biohydrogels as electrolyte system}

After discarding chitosan hydrogels as electrolyte due to its extremely poor mechanical integrity, the electrochemical response of PEDOT in each of the remaining three biohydrogels was investigated to determine their effectiveness as electrolytic media. Fig. S5a-S5c $\dagger$ display cyclic voltammograms recorded after several consecutive redox scans for $\kappa$-carrageenan, Naalginate and gelatin electrolyte systems, respectively, using a three-electrode configuration. PBS was used as control electrolyte system (Fig. S5d $\dagger$ ). These voltammograms have been used to evaluate the electroactivity of PEDOT (Fig. 1a and b), which corresponds to the similarity between the cathodic and anodic areas in the voltammograms (i.e. ratio between the reduction and oxidation charges, $\left.Q_{\text {red }} / Q_{\text {ox }}\right)$. The electroactivity increases with the number of redox cycles indicating that the ability to exchange charge reversibly improves and stabilizes after fifty consecutive oxidation-reduction cycles. Specifically, after the tenth cycle, the $Q_{\text {red }} / Q_{\text {ox }}$ ratio is higher than 0.90 for the three biohydrogel-based electrolytes (Fig. 1b). Comparison of the current densities reached at the reversal potential (i.e. $1.0 \mathrm{~V}$ in Fig. S5a-S5d $\dagger$ ) indicates that the amount of oxidized molecules is similar for $\kappa$-carrageenan, Na-alginate and PBS, whereas it is lower for gelatin-based electrolyte. Moreover, the electrochemical stability of PEDOT also changes with the electrolyte system (Fig. 1c). After five-hundred redox cycles, the loss of electrochemical activity (LEA, eqn (1)) was slightly lower in $\mathrm{k}$ carrageenan $(13 \%)$ than in Na-alginate and gelatin $(18 \%$ and $16 \%$, respectively). Moreover, the amount of charge stored by PEDOT electrodes varies depending on the electrolyte (Fig. 1a): $\kappa$-carrageenan $>$ Na-alginate $>$ PBS $>$ gelatin.

Results indicate that the compact morphology of gelatin hydrogel hinders the access and escape of dopant ions during the oxidation and reduction processes of PEDOT, which reduces both the amount of charge stored and the current density. Furthermore, the response of PEDOT in both $\kappa$-carrageenan and Na-alginate hydrogel-based electrolytes is similar, indicating that the interconnecting network of both systems enables ion transport. Finally, the electrochemical performance of PEDOT in PBS electrolyte is worse in comparison to $\kappa$-carrageenan and Na-alginate based devices. More specifically, the electroactivity and LEA values obtained in PBS are lower and higher, respectively, than those reached using such two biohydrogels. These features indicate that the swelling and shrinkage of PEDOT electrodes during redox processes are enhanced in PBS electrolyte, inducing a faster and more severe mechanical degradation of the ECP that, in turn, affects its electrochemical properties.

Fig. $1 \mathrm{~d}$ and e show the variation of both the coulombic efficiency (eqn (4)) and the SC (eqn (2) and (3)), respectively, against the current density for the three biopolymer hydrogel-based OESC systems. Data derive from the corresponding GCD curves 
(a)

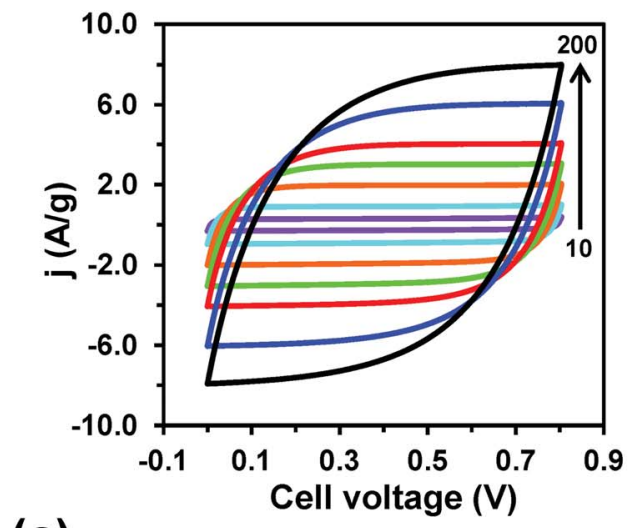

(c)

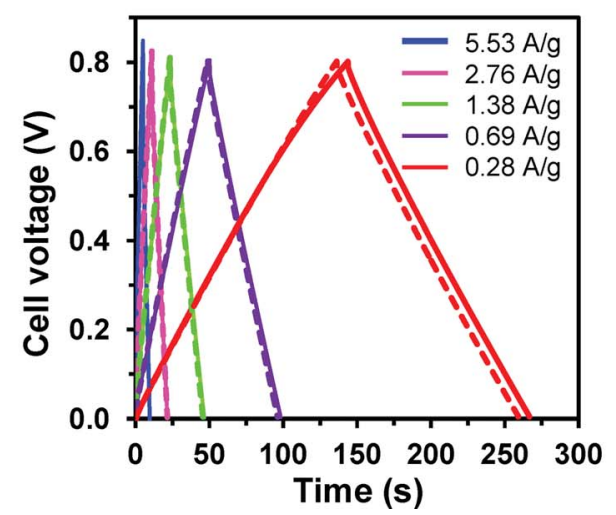

(b)

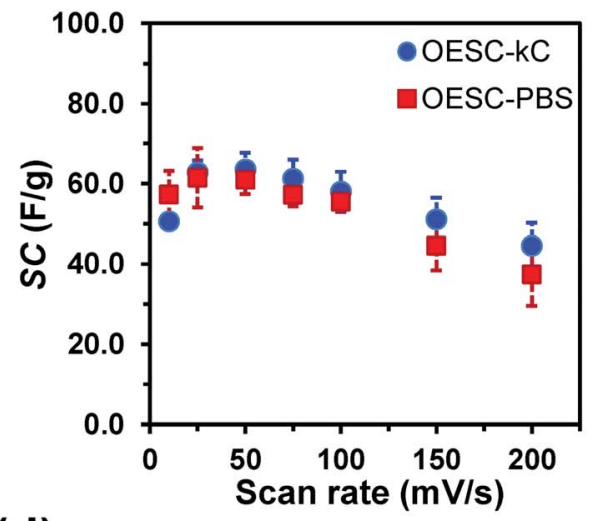

(d)

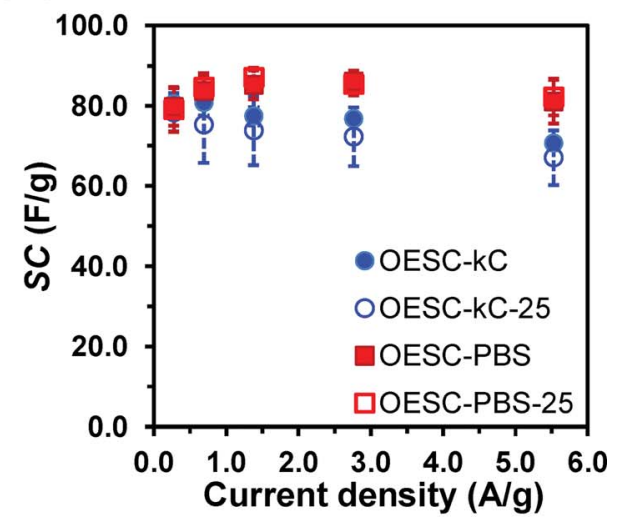

Fig. 3 Electrochemical characterization (two-electrode configuration) of OESC- $\kappa$ C devices, which present both CE and WE made of PEDOT and $\kappa$-carrageenan hydrogel as electrolyte. (a) Cyclic voltammograms recorded from 0.0 to $0.8 \mathrm{~V}$ after five consecutive redox cycles at scan rates of $10,25,50,75,100,150$ and $200 \mathrm{mV} \mathrm{s}^{-1}$ (b) SC values derived from the cyclic voltammograms displayed in (a) (see eqn (2) and (3)). (c) GCD curves recorded from 0.0 to $0.8 \mathrm{~V}$ at different current densities. Solid lines correspond to the first charge-discharge cycle, while the dashed ones correspond to the twenty-fifth cycle. (d) SC values derived from the GCD curves displayed in (c) (see eqn (2) and (3)). SC values in (b) and (d) are compared with those obtained for the OESC-PBS control device (Fig. S7 †). In (d) empty symbols refer to the SC values determined for the twentyfifth charge-discharge cycle.

(Fig. S6 $\dagger$ ). As it can be seen, the triangular shape of the GCD curves and SC values are better defined and higher, respectively, for OESC- $\kappa \mathrm{C}$ than for OESC-NaAlg and OESC-Gel, independently of the current density used. In addition, after 25 chargedischarge processes, the SC determined for OESC- $\mathrm{\kappa C}$ remained higher than $96 \%$ for all the considered current densities (Fig. S6 $\dagger$ ). For example, for the current density of $1.38 \mathrm{~A} \mathrm{~g}^{-1}$, the SC only decreased $0.98 \%$, evidencing excellent cycling stability.

Overall, these results reflect that $\kappa$-carrageenan is the most suitable biohydrogel to perform as electrolyte due to the appropriate combination of properties: mechanical stability, ease of preparation, lack of water leaking, and good medium for the electrochemical response of PEDOT. Accordingly, investigations displayed in next sections are exclusively focused on OESC-кC devices (Fig. 2).

\section{OESC- $\kappa \mathrm{C}$ energy storage device: capacitive performance}

Specific capacitance. The capacitive behavior of OESC-кC was investigated by CV and GCD curves (Fig. 3). Results have been compared with those obtained for an OESC device with
PBS as electrolytic medium (OESC-PBS), which has been used as a control (Fig. $S 7 \dagger$ ).

Cyclic voltammograms recorded for the OESC- $\kappa \mathrm{C}$ device (Fig. 3a) from 0.0 to $0.8 \mathrm{~V}$ display an almost ideal rectangular shape for scan rates up to $200 \mathrm{mV} \mathrm{s}^{-1}$ (Fig. 3a), which is indicative of a good capacitive performance and low contact resistance. This effect is less pronounced for OECS-PBS (Fig. S7a †). Furthermore, the current density at the reversal and initial potential (i.e. anodic and cathodic current density, respectively) varies linearly with the scan rate (Fig. S8 †), evidencing that the electrochemical processes at OESC- $\mathrm{KC}$ and OESC-PBS are not controlled by diffusion. ${ }^{54,55}$ On the other hand, except for the lowest scan rate, the SC values (eqn (2) and (3)) obtained for OESC- $\kappa \mathrm{C}$ are slightly higher than those determined for OESC-PBS (Fig. $3 \mathrm{~b}$ and Table S1†).

GCD curves for OESC- $\kappa \mathrm{C}$ devices exhibited a typical triangular shape with a small voltage drop at the beginning of the discharging step (Fig. 3c). In general, PEDOT electrodes display symmetric charge-discharge curves that correspond to almost ideal capacitive properties: high coulombic efficiency and good reversibility. ${ }^{14,16,18}$ These features are observed for OESC- $\kappa \mathrm{C}$ 
(a)

(c)

(e)
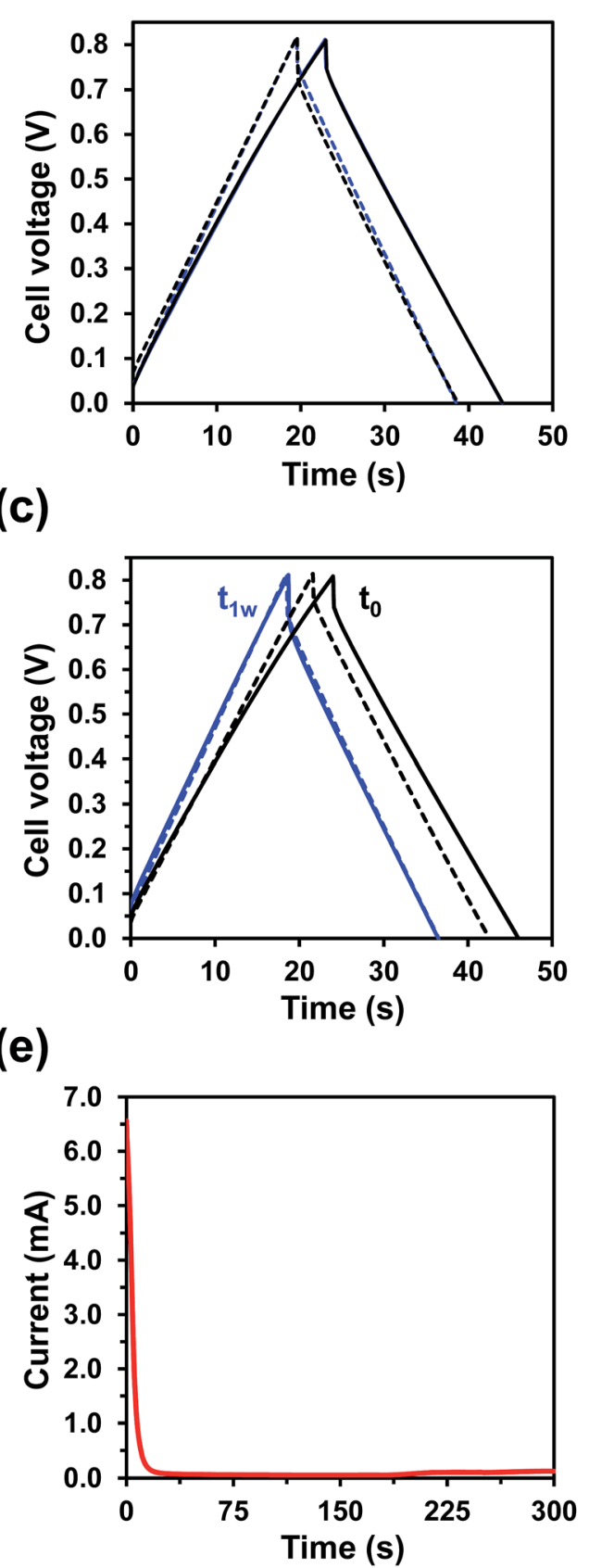

(b)

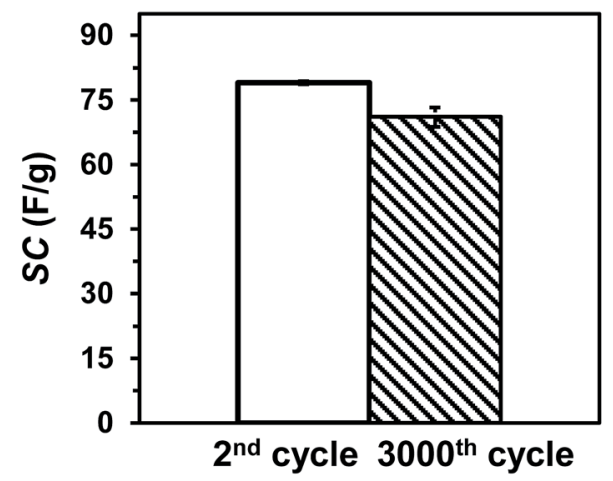

(d)

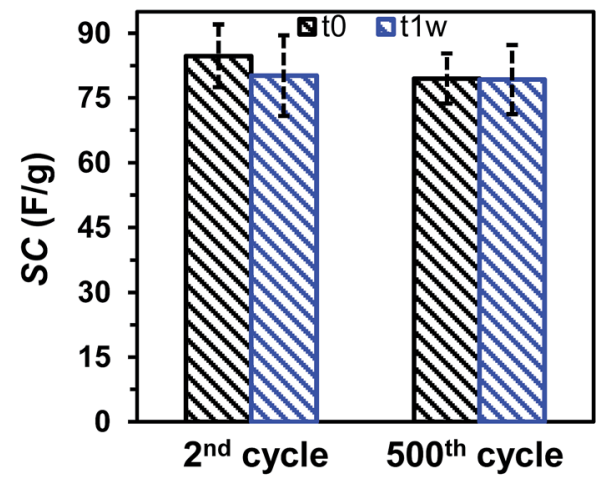

(f)

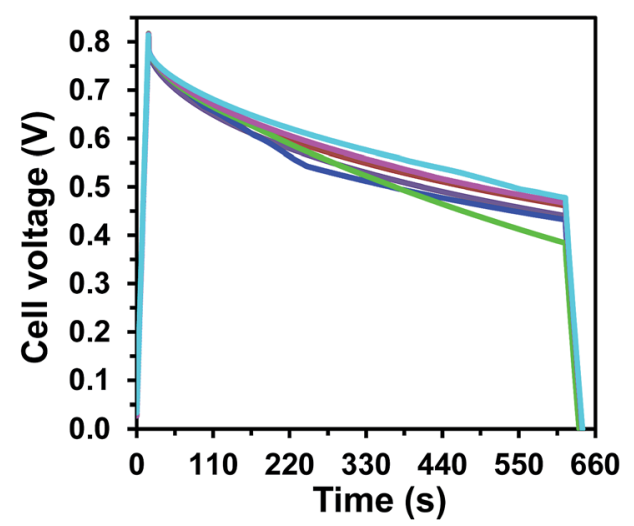

Fig. 4 Cycling stability of OESC- $\kappa \mathrm{C}$ devices, which present both CE and WE made of PEDOT and $\kappa$-carrageenan hydrogel as electrolyte. (a) GCD curves recorded from 0 to $0.8 \mathrm{~V}$ at $1.38 \mathrm{~A} \mathrm{~g}^{-1}$ (two repetitions). Solid lines correspond to the second cycle, while the dashed ones correspond to the three-thousandth cycle. (b) SC values calculated with the data derived from (a). (c) GCD curves recorded from 0 to $0.8 \mathrm{~V}$ at $1.38 \mathrm{~A} \mathrm{~g}^{-1}$ for devices tested just as prepared $\left(t_{0}\right)$ and after one week of their preparation $\left(t_{1 w}\right)$. Solid lines correspond to the first cycle, while the dashed ones correspond to the five-hundredth cycle. (d) SC values calculated with the data derived from (c). (e) Leakage current curve for OESC- $\kappa \mathrm{C}$ charged

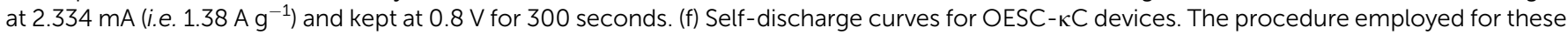
assays is described in the Methods section.

devices, which results in SC values (eqn (2) and (3)) that range from 70 to $81 \mathrm{~F} \mathrm{~g}^{-1}$ for the second charge-discharge cycle (Fig. $3 \mathrm{~d}$ and Table $\mathrm{S} 2 \dagger$ ). Although these SC values are similar to those obtained for the OESC-PBS device at low current densities (Fig. S7 $\dagger$ ), they tend to decrease for higher current densities, possibly due to slower ion movement and electrolyte diffusion in the biohydrogel-based electrolyte. In contrast, SC values for the OESC-PBS device do not present significant changes with the current density (Fig. 3d). On the other hand, after 25 consecutive charge-discharge cycles, OESC- $\kappa \mathrm{C}$ devices displayed an excellent SC retention (i.e. higher than 92\%) for all tested current densities (Fig. 3d). For example, the system was 
(a)

able to retain $c a .95 \%$ of the highest SC value, which was obtained at $1.38 \mathrm{~A} \mathrm{~g}^{-1}$ (Table $\mathrm{S} 2 \dagger$ ). As it was expected, the stability of the OESC- $\mathrm{KC}$ device is slightly lower than that of the OESCPBS one (Fig. $3 \mathrm{~d}$ and Table S2 $\dagger$ ). This should be attributed to the degradative effects produced by consecutive charge-discharge cycles in the hydrogel structure.

Overall, results displayed in this sub-section confirm that the OESC based on PEDOT electrodes and $\kappa$-carrageenan as electrolyte exhibits a good supercapacitor response. The presence of the solid electrolyte does not affect negatively the PEDOT capacitive behavior, bringing advantageous characteristics to the final packaged cell for practical applications.

Lifetime stability. Cyclability (i.e. from hundreds to thousands of cycles) is an important requirement for practical supercapacitor applications. In order to evaluate this property, as-prepared OESC- $\kappa \mathrm{C}$ devices were submitted to three-thousand consecutive GCD cycles at a constant current density of $1.38 \mathrm{~A}$ $\mathrm{g}^{-1}$ from 0 to $0.8 \mathrm{~V}$, results being summarized in Fig. $4 \mathrm{a}$ and $\mathrm{b}$. After such amount of cycles, the SC decrease from $79.1 \pm 0.4 \mathrm{~F}$ $\mathrm{g}^{-1}$ to $71.1 \pm 2.2 \mathrm{~F} \mathrm{~g}^{-1}$, which represents a variation of only $\sim 10 \%$ (i.e. $90 \%$ of retention). This slight reduction in the SC values for OESC- $\kappa \mathrm{C}$ has been associated to the swelling and shrinkage phenomena experienced by PEDOT during oxidation (b)

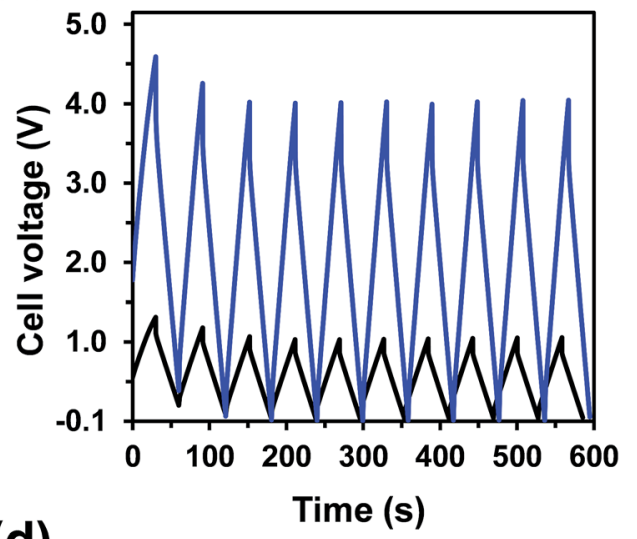

(d)

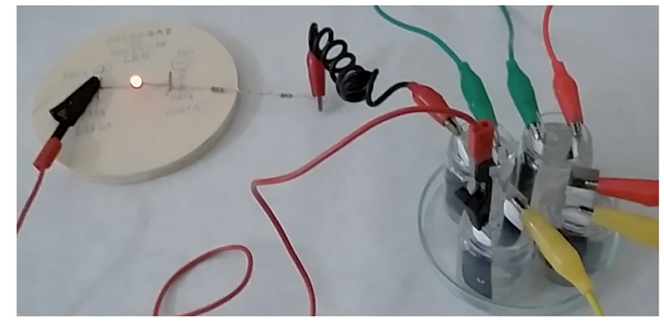

and reduction processes, respectively. Thus, consecutive doping-dedoping steps induce structural changes in PEDOT electrodes, which evolve from a porous morphology to a more compact one. The increasing compactness of PEDOT hinders the access and escape of dopant ions during the oxidation and reduction processes, respectively, until the equilibrium is reached.

Moreover, to determine the lifetime stability of OESC-кC, the same assay was repeated after one week of the device preparation. Fig. $4 \mathrm{c}$ and d compare the results obtained for as asprepared OESC- $\kappa \mathrm{C}$ devices $\left(t_{0}\right)$ with those obtained for devices prepared one week before $\left(t_{1 \mathrm{w}}\right)$. In this series of as prepared devices, the SC decrease from $85 \pm 7 \mathrm{~F} \mathrm{~g}^{-1}$ to $80 \pm 6 \mathrm{~F} \mathrm{~g}^{-1}$ after consecutive GCD cycles at a constant current density of $1.38 \mathrm{~A}$ $\mathrm{g}^{-1}$ from 0 to $0.8 \mathrm{~V}$, which represents a variation of only $\sim 6 \%$ (i.e. $94 \%$ of retention). For the devices that were characterized one week after their preparation, the SC values corresponding to the second $\left(80 \pm 9 \mathrm{~F} \mathrm{~g}^{-1}\right)$ and five-hundredth $\left(79 \pm 8 \mathrm{~F} \mathrm{~g}^{-1}\right)$ charge-discharge cycle differ by only $\sim 1 \%$ (i.e. $99 \%$ of retention) and, more interestingly, they resemble to those determined for the as-prepared device after five-hundred cycles (79 \pm $6 \mathrm{~F} \mathrm{~g}^{-1}$ ), confirming that the structure of PEDOT is stabilized. In addition to this excellent cycling stability, OESC- $\kappa \mathrm{C}$ devices, 
when properly closed (Fig. S9a $\dagger$ ), exhibit no water loss or evaporation, which further ensures its application.

Leakage current and self-discharge response. The leakage current is the stable parasitic current expected when the capacitor is held indefinitely on charge at the rated voltage. ${ }^{56}$ OESC- $\kappa \mathrm{C}$ devices were charged to $0.8 \mathrm{~V}$ at $2.334 \mathrm{~mA}$ (i.e. $1.38 \mathrm{~A}$ $\left.\mathrm{g}^{-1}\right)$, and kept at that potential for 5 minutes, while recording the current data through the OESC-кC (i.e. leakage current). Fig. 4e shows how the current quickly decreases to a minimum value of $51.2 \mu \mathrm{A}$, staying at that value for the remaining time. This relatively small leakage current is associated to a good stability of OESC- $\kappa \mathrm{C}$ devices, which is crucial for energy storage applications.

On the other hand, self-discharge is understood as the voltage drop on a charged capacitor after a set period of time. Such loss of charge, which may result in dysfunctional conditions, affect the electrochemical response of the capacitor by lowering the power and energy densities. Therefore, the rate of self-discharge has a major practical significance when assessing the performance and specifications of any energy storage device. Self-discharge is influenced by different factors, such as the chemistry and electrochemistry of the system, the purity of reagents and electrolyte and, mainly, the temperature. ${ }^{57}$

The self-discharging characteristics of OESC- $\kappa \mathrm{C}$ were determined by considering the following three-step test: (i) the OESC$\kappa \mathrm{C}$ was charged to $0.8 \mathrm{~V}$ at $2.334 \mathrm{~mA}$; (ii) it was kept at $1 \times 10^{-11}$ $\mathrm{mA}$ for $10 \mathrm{~min}$ (self-discharging); and (iii) the device was discharged to $0.2 \mathrm{~V}$ at $-1 \mathrm{~mA}$. Fig. $4 \mathrm{f}$ evidences how, for most of the cases, the voltage of OESC- $\kappa \mathrm{C}$ ended above $0.4 \mathrm{~V}$ after the set period of time. Although elucidation of the mechanisms and driving forces that control self-discharge is beyond the scope of this study, results validate the low self-discharging tendency of OESC- $\kappa \mathrm{C}$ devices, at least in the short term, ensuring specific practical applications.

Practical application using a LED bulb. Four OESC- $\kappa \mathrm{C}$ devices were assembled in series (Fig. 5a, S9b and S9c $\dagger$ ) and subjected to the following GCD measurements: 10 chargedischarge cycles for 30 seconds at $1.38 \mathrm{~A} \mathrm{~g}^{-1}$. Consequently, by assembling four devices the voltage window increases up to $4 \mathrm{~V}$, while curves retain the symmetric shape, and thus the capacitance performance of individual OESC- $\kappa \mathrm{C}$ (Fig. 5b). In a similar

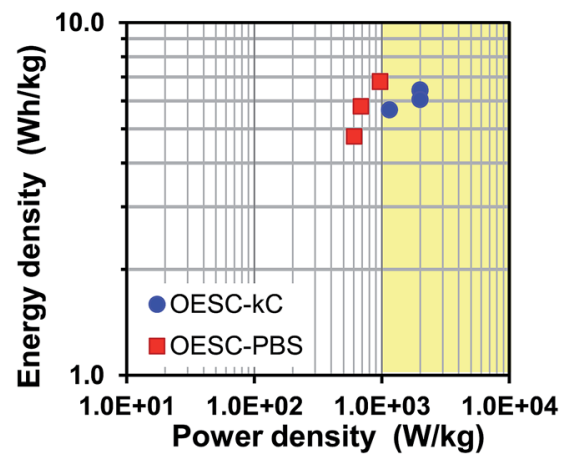

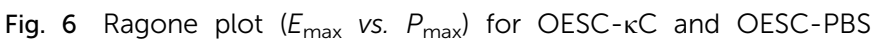
devices. way, self-discharging curves for the assembled devices decrease from $4 \mathrm{~V}$ to $c a$. $1.5 \mathrm{~V}$ after 10 minutes (Fig. 5c).

To further confirm the suitability of OESC- $\kappa \mathrm{C}$ for practical applications, the assembled devices were used to power a red LED (lowest working potential 1.65-1.7 V, Fig. 5d). For this purpose, the assembled system was charged to $4.0 \mathrm{~V}$ at $1.38 \mathrm{~A}$ $\mathrm{g}^{-1}$ and connected to the LED and two resistances in series (2 $\times 100 \Omega$ ), while recording the time the LED was lighted. For comparison purposes, this procedure was also applied to four assembled OESC-PBS devices, and repeated five times for each system (videos are provided in the ESI $\dagger$ ). Results indicated that when using OESC- $\kappa \mathrm{C}$ the LED was lit for a longer time $(35 \pm 5 \mathrm{~s})$ than in the case of control OESC-PBS $(20 \pm 4 \mathrm{~s})$.

Ragone plot. Energy $\left(E_{\max }\right.$; eqn (5)) and power ( $P_{\max }$; eqn (6)) densities, which are important parameters for assessing the supercapacitor performance of energy storage devices, are represented in Ragone plots (i.e. $E_{\max }$ against $P_{\max }$ ) to enable the comparison of different systems. ${ }^{58}$ Thus, these plots indicate how much energy is available (vertical axis) and how quickly it can be delivered (horizontal axis). The Ragone plot obtained for OESC- $\kappa$ C and OESC-PBS (Fig. 6) evidences that the two systems display similar energy density $\left(6.1 \pm 0.4\right.$ and $5.8 \pm 1.0 \mathrm{~W} \mathrm{~h} \mathrm{~kg}^{-1}$, respectively), whereas the power density is higher for the former than for the latter $\left(1.7 \pm 0.5\right.$ and $0.8 \pm 0.2 \mathrm{~kW} \mathrm{~kg}^{-1}$, respectively).

Considering that energy and power densities of supercapacitors for power applications are comprised in the interval between 1-9 $\mathrm{W} \mathrm{h} \mathrm{kg}^{-1}$ and $1-10 \mathrm{~kW} \mathrm{~kg}^{-1}$, respectively, ${ }^{59}$ the OESC $-\kappa \mathrm{C}$ system behaves as a good candidate for such application. It is of highly importance to remember that data in the Ragone plot (Fig. 6) has to be considered carefully since it is based only on the total mass of active PEDOT electrodes, in accordance to the methodology described in previous works. ${ }^{56}$ However, as stated by Stoller and Ruoff, ${ }^{\mathbf{3 8}} P_{\max }$ should be computed by considering the total mass of the device, which also includes dead components and the electrolyte.

\section{Improving the OESC- $\kappa \mathrm{C}$ energy storage device}

Different strategies could be considered to obtain OESC- $\kappa \mathrm{C}$ devices with higher energy and power densities. On the one hand, a relatively simple strategy to enhance the power density is the design of supercapacitors with compact cell architectures (i.e. reducing the electrolyte volume) to decrease the total mass of the device. On the other hand, the energy density improves by increasing the SC of the device, which can be accomplished by properly modifying the electrode material to display larger specific surface area, higher conductivity, and better pore size distribution to favour ion diffusion. For example, the SC of a symmetrical pseudocapacitor improved substantially after modifying the PEDOT-PSS electrodes with $\mathrm{RuO}_{2} \cdot x \mathrm{H}_{2} \mathrm{O} .^{60}$ However, although these modifications are known to have satisfactory effects on the performance of the device, they are time-demanding and require additional synthetic routes to alter the properties of PEDOT electrodes. ${ }^{25}$ 
(a)

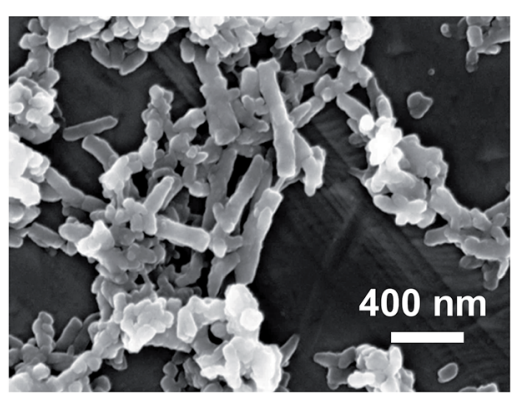

(c)

(e)
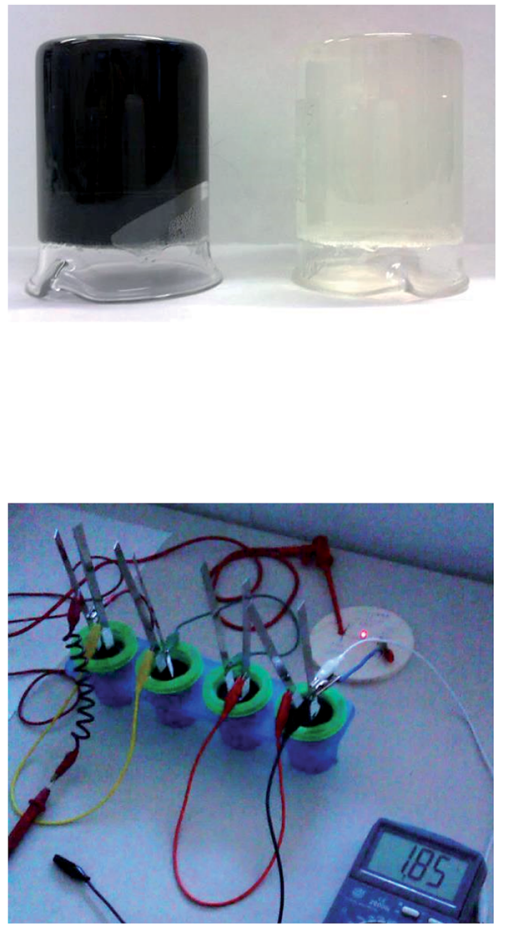

(b)

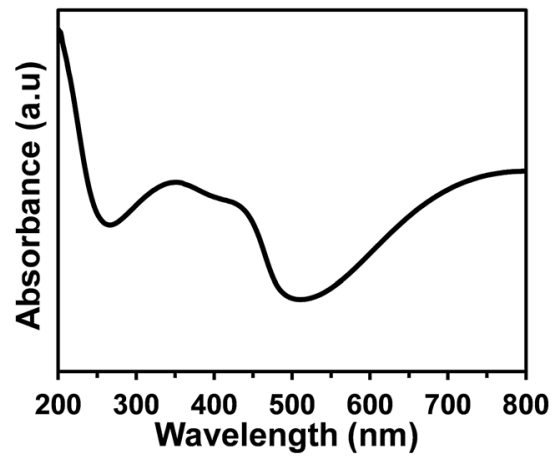

(d)

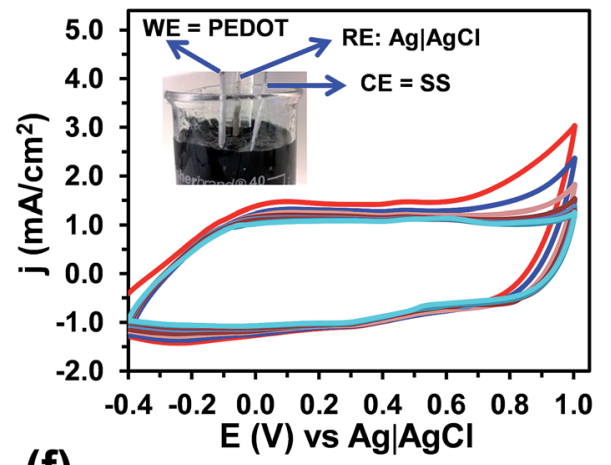

(f)

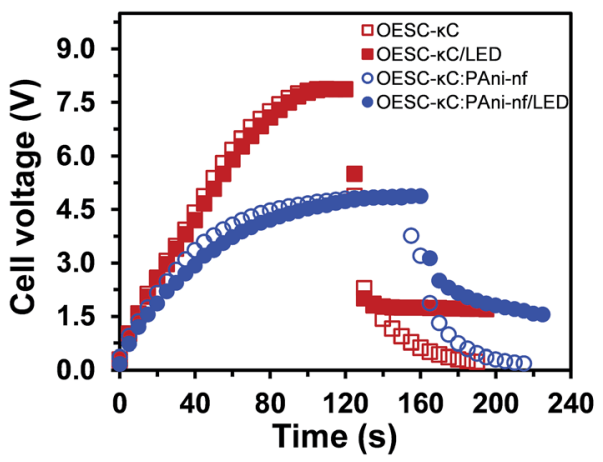

Fig. 7 (a) SEM image of PAni-nfs (100 kX). (b) UV-vis spectrum for a diluted solution of as-prepared PAni-nfs (1/100). (c) $\kappa$-Carrageenan hydrogel with (left) and without (right) PAni-nfs. (d) Cyclic voltammograms for PEDOT in three-electrode configuration recorded from -0.4 to $1.0 \mathrm{~V}$ at a scan rate of $50 \mathrm{mV} \mathrm{s}^{-1}$ using $\kappa$-carrageenan hydrogel loaded with Pani-nfs as electrolyte media. (e) Four OESC- $\kappa C$ :PAni-nfs assembled in series

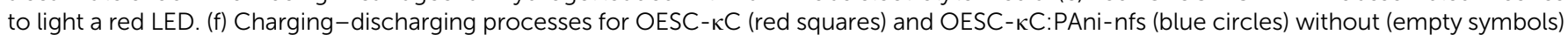
and with LED (filled symbols).

In addition to the previous two strategies, another interesting action to raise the SC is based on tailoring the electrolyte element. Thus, the introduction of redox additives into conventional aqueous electrolytes to induce electrochemical activity and reversibility has been described in recent studies. ${ }^{61-63}$ This modification contributes to the pseudocapacitance of the system through additional redox processes between the electrode and the added electrolyte. Similarly, the energy storage performance of solid-state supercapacitors has been improved adding redox additives to the polymeric electrolyte..$^{54,64,65}$

Inspired by some of these strategies, the performance of the OESC- $\kappa \mathrm{C}$ energy storage device has been re-examined after the incorporation of PAni nanofibers (PAni-nfs) to the electrolyte system. The length and diameter of PAni-nfs, which were prepared using a previously described procedure (ESI $\dagger$ ), ${ }^{66-68}$ are $\sim 400$ and $\sim 120 \mathrm{~nm}$, respectively, as determined from SEM micrographs (Fig. 7a). On the other hand, the doped state of the ECP was corroborated by examining polaronic bands in the UVvis spectrum of PAni-nfs solution (Fig. 7b).

OESC- $\kappa \mathrm{C}$ devices incorporating PAni-nfs in the hydrogel electrolyte (OESC- $\kappa \mathrm{C}:$ PAni-nfs) were fabricated as is described in the Methods section. Specifically, PAni-nfs were introduced in the $\kappa$-carrageenan hydrogel before the gelation step, thus rendering a hydrogel-based electrolyte with redox additives. Fig. $7 \mathrm{c}$ compares the unloaded and loaded $\kappa$-carrageenan hydrogels. 
Cyclic voltammograms recorded for PEDOT (three-electrode configuration) using $\kappa$-carrageenan hydrogel with PAni-nfs as electrolyte are displayed in Fig. 7d. Comparison with the voltammograms displayed in Fig. S5a, $\uparrow$ which correspond to the unloaded system, indicates that the presence of PAni-nfs in the hydrogel-based electrolyte does not affect the electrochemical response of PEDOT. This conclusion is supported by Fig. S10, $\dagger$ which represents the variation of the oxidation and reduction charges ( $Q_{\text {ox }}$ and $Q_{\text {red }}$, respectively), the $Q_{\text {red }} / Q_{\text {ox }}$ ratio and the LEA against the number of consecutive oxidation-reduction cycles.

Four OESC- $\kappa \mathrm{C}:$ PAni-nfs devices were assembled to power a red LED (lowest working potential 1.65-1.7 V). For this test, the system was charged at $\sim 0.75 \mathrm{~A} \mathrm{~g}^{-1}(1.28 \mathrm{~mA})$ to a potential higher than $4.0 \mathrm{~V}$ for at least 100 seconds, and then connected in series with a resistance $(1 \mathrm{k} \Omega)$. During the charging-discharging process the potential was monitored every five seconds. After this, the charging-discharging sequence was repeated connecting also the LED to the circuit (Fig. 7e). For comparison, this procedure, which differs from that discussed in the previous section, was also applied to four assembled OESC-кC devices (Fig. S11†).

Inspection of the results displayed in Fig. $7 \mathrm{f}$ indicates that during the discharging step the potential drop is lower for the

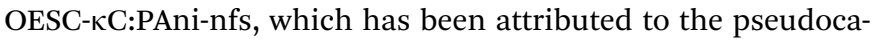
pacitance provided by PAni-nfs. Thus, the energy retention

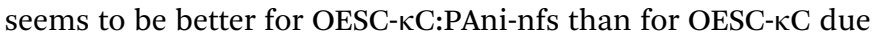
to the high SC of PAni-nfs. The OESC- $\kappa \mathrm{C}:$ PAni-nfs device is able to power the LED during 45 seconds, whereas for the OESC- $\kappa \mathrm{C}$ control system the LED was on during $\sim 30$ seconds. This preliminary but very promising result suggests that the integration of $\kappa$-carrageenan and PAni-nfs is a good strategy for the fabrication of effective and compact OESCs based on biopolymer hydrogels. Our most immediate objective consists on the optimization of OESC- $\kappa \mathrm{C}:$ PAni-nfs devices, which will be addressed by examining the role played by the distribution of PAni-nfs into the biohydrogel, incorporating auxiliary redox agents and improving the organic electrodes to eliminate the use of stainless steel collectors, to enhance their performance.

\section{Conclusions}

An OESC has been designed in an effort to evolve towards greener, more sustainable and more compact devices. For that, both electrodes have been prepared as PEDOT thin-films, while the electrolyte was based on a biopolymeric hydrogel. Among the tested systems, $\kappa$-carrageenan biohydrogel has been found to be the most appropriated as electrolytic medium because of its mechanical stability, ease of preparation, lack of water leaking, and ability to maintain a good response of PEDOT electrodes. Indeed, results indicate that replacement of aqueous electrolyte by $\kappa$-carrageenan hydrogel does not affect the electrochemical response of PEDOT and the capacitance response of fabricated OESC devices. Thus, OESC devices prepared using $\kappa$-carrageenan hydrogel as electrolytic medium have shown good capacitor response, excellent cycling stability, small leakage current, low self-discharging tendency, and no water loss or evaporation. Finally, the practical application of OESC$\kappa \mathrm{C}$ devices has been illustrated by powering a red LED.

On the other hand, preliminary assays have been carried out adding PAni-nfs, which act as a redox additive, into the biohydrogel. Results obtained for OESC- $\kappa \mathrm{C}:$ PAni-nfs devices are very promising, future developments being very convenient. These will deal with preparing a more advanced functional OESC- $\kappa \mathrm{C}$ system, which will include among others modifications, the use of auxiliary redox agents and flexible organic electrodes.

\section{Acknowledgements}

Authors are indebted to supports from MICINN and FEDER (MAT2012-34498), the University of Regensburg and the DFG (DI 1748/3-1). M. M. P.-M. thanks financial support through a FPI-UPC grant and a mobility DAAD Research Grant for Doctoral Candidates and Young Academics and Scientists (German Academic Exchange Service, DAAD). D. D. D. thanks the DFG for the Heisenberg Professorship Award. Support for the research of C.A. was received through the prize "ICREA Academia" for excellence in research funded by the Generalitat de Catalunya.

\section{References}

1 P. Simon and Y. Gogotsi, Nat. Mater., 2008, 7, 845.

2 J. Deng, T. Xiong, F. Xu, M. Li, C. Han, Y. Gong, H. Wang and Y. Wang, Green Chem., 2015, 17, 4053.

3 D. H. Seo, S. Pineda, S. Yick, J. Bell, Z. J. Han and K. K. Ostrikov, Green Chem., 2015, 17, 2164.

4 W. Qian, Z. Chen, S. Cottingham, W. A. Merrill, N. A. Swartz, A. M. Goforth, T. L. Clare and J. Jiao, Green Chem., 2012, 14, 371.

5 D. Aradilla, F. Estrany, E. Armelin and C. Alemán, Thin Solid Films, 2012, 520, 4402.

6 G. Yu, X. Xie, L. Pan, Z. Bao and Y. Cui, Nano Energy, 2013, 2, 213.

7 L. Wang, X. Feng, L. Ren, Q. Piao, J. Zhong, Y. Wang, H. Li, Y. Chen and B. Wang, J. Am. Chem. Soc., 2015, 137, 4920.

8 K. Naoi, W. Naoi, S. Aoyagi, J.-i. Miyamoto and T. Kamino, Acc. Chem. Res., 2013, 46, 1075.

9 R. Ranjusha, K. M. Sajesh, S. Roshny, V. Lakshmi, P. Anjali, T. S. Sonia, A. S. Nair, K. R. V. Subramanian, S. V. Nair, K. P. Chennazhi and A. Balakrishnan, Microporous Mesoporous Mater., 2014, 186, 30.

10 F. Su and M. Miao, Electrochim. Acta, 2014, 127, 433.

11 B. E. Conway, in Electrochemical Supercapacitors, Springer Science+Buisness Media, New York, USA, 1999, ch. 10, pp. 221-257.

12 M. Mastragostino, C. Arbizzani and F. Soavi, Solid State Ionics, 2002, 148, 493.

13 J. F. Mike and J. L. Lutkenhaus, J. Polym. Sci., Part B: Polym. Phys., 2013, 51, 468.

14 D. Aradilla, F. Estrany and C. Alemán, J. Phys. Chem. C, 2011, 115, 8430. 
15 J. Wang, Z. Wu, H. Yin, W. Li and Y. Jiang, RSC Adv., 2014, 4, 56926.

16 D. Aradilla, D. Azambuja, F. Estrany, M. T. Casas, C. A. Ferreira and C. Alemán, J. Mater. Chem., 2012, 22, 13110.

17 X. Bai, X. Hu and S. Zhou, RSC Adv., 2015, 5, 43941.

18 Y. Shi, L. Peng and G. Yu, Nanoscale, 2015, 7, 12796.

19 Y. Zhao, B. Liu, L. Pan and G. Yu, Energy Environ. Sci., 2013, 6, 2856.

$20 \mathrm{~W}$. Zhao, L. Glavas, K. Odelius, U. Edlund and A.-C. Albertsson, Chem. Mater., 2014, 26, 4265.

21 J. F. Mike and J. L. Lutkenhaus, ACS Macro Lett., 2013, 2, 839.

22 C. Chen, X. Fu, T. Ma, W. Fan, Z. Wang and S. Miao, J. Appl. Polym. Sci., 2014, 131, 40814.

23 L. Pan, G. Yu, D. Zhai, H. R. Lee, W. Zhao, N. Liu, H. Wang, B. C.-K. Tee, Y. Shi, Y. Cui and Z. Bao, Proc. Natl. Acad. Sci. U. S. A., 2012, 109, 9287.

24 Y. Shi, L. Pan, B. Liu, Y. Wang, Y. Cui, Z. Bao and G. Yu, J. Mater. Chem. A, 2014, 2, 6086.

25 C. Zhao and W. Zheng, Frontiers in Energy Research, 2015, 3, 23.

26 H. Huang, X. Zeng, W. Li, H. Wang, Q. Wang and Y. Yang, J. Mater. Chem. A, 2014, 2, 16516.

27 S. Kumar, Z. Hicks and T. Viswanathan, ACS Sustainable Chem. Eng., 2015, 3, 2194.

28 S. K. Ramasahayam, A. L. Clark, Z. Hicks and T. Viswanathan, Electrochim. Acta, 2015, 168, 414.

29 N. Zhang, W. Zhou, Q. Zhang, P. Luan, L. Cai, F. Yang, X. Zhang, Q. Fan, W. Zhou, Z. Xiao, X. Gu, H. Chen, K. Li, S. Xiao, Y. Wang, H. Liu and S. Xie, Nanoscale, 2015, 7, 12492.

30 U. B. Nasini, V. G. Bairi, S. K. Ramasahayam, S. E. Bourdo, A. U. Shaikh and T. Viswanathan, J. Power Sources, 2013, 250, 257.

31 M. R. Lukatskaya, O. Mashtalir, C. E. Ren, Y. Dall'Agnese, P. Rozier, P. L. Taberna, M. Naguib, P. Simon, M. W. Barsoum and Y. Gogotsi, Science, 2013, 341, 1502.

32 X. Lu, M. Yu, G. Wang, Y. Tong and Y. Li, Energy Environ. Sci., 2014, 7, 2160.

33 N. Buchtová, A. Guyomard-Lack and J. L. Bideau, Green Chem., 2014, 16, 1149.

34 N. A. Choudhury, S. Sampath and A. K. Shukla, Energy Environ. Sci., 2009, 2, 55.

35 J. Liu, Q. Zhang, Z.-Y. Wu, J.-H. Wu, J.-T. Li, L. Huang and S.-G. Sun, Chem. Commun., 2014, 50, 6386.

36 M. Rosi, F. Iskandar, M. Abdullah and Khairurrijal, Int. J. Electrochem. Sci., 2014, 9, 4251.

37 N. A. Choudhury, S. Sampath and A. K. Shukla, J. Electrochem. Soc., 2008, 155, A74.

38 M. D. Stoller and R. S. Ruoff, Energy Environ. Sci., 2010, 3, 1294.

39 Y. Aiping, V. Chabot and J. Zhang, in Electrochemical Supercapacitors for Energy Storage and Delivery Fundamentals and Applications, CRC Press, Boca Raton, USA, 2013, ch. 7, pp. 277-316.
40 M. Kaempgen, C. K. Chan, J. Ma, Y. Cui and G. Gruner, Nano Lett., 2009, 9, 1872.

41 Y. Xu, J. Wang, W. Sun and S. Wang, J. Power Sources, 2006, 159, 370.

42 J.-H. Huang and C.-W. Chu, Electrochim. Acta, 2011, 56, 7228.

43 Y. Li, B. Wang, H. Chen and W. Feng, J. Power Sources, 2010, $195,3025$.

44 K. Lota, V. Khomenko and E. Frackowiak, J. Phys. Chem. Solids, 2004, 65, 295.

45 I. Shown, A. Ganguly, L.-C. Chen and K.-H. Chen, Energy Sci. Eng., 2015, 3, 2.

46 Y. Zhang and K. S. Suslick, Chem. Mater., 2015, 27, 7559.

47 B. H. Jones, K.-Y. Cheng, R. J. Holmes and T. P. Lodge, Macromolecules, 2012, 45, 599.

48 Y. Han, B. Ding, H. Tong and X. Zhang, J. Appl. Polym. Sci., 2011, 121, 892.

49 R. Mukkabla, M. Deepa and A. K. Srivastava, $R S C A d v ., 2015$, 5, 99164.

50 R. Mukkabla, M. Deepa and A. K. Srivastava, ChemPhysChem, 2015, 16, 3242.

51 S. Lehtimäki, M. Suominen, P. Damlin, S. Tuullanen, C. Kvarnström and D. Lupo, ACS Appl. Mater. Interfaces, 2015, 7, 22137.

52 S. Ahmad, J.-H. Yum, Z. Xianxi, M. Grätzel, H.-J. Butt and M. K. Nazeeruddin, J. Mater. Chem., 2010, 20, 1654.

53 J. Chao, Z. Xie, X. B. Duan, Y. Dong, Z. Wang, J. Xu, B. Liang, B. Shan, J. Ye, D. Chen and G. Shen, CrystEngComm, 2012, 14, 3163.

54 L. Chen, Y. Chen, J. Wu, J. Wang, H. Bai and L. Li, J. Mater. Chem. A, 2014, 2, 10526.

55 A. J. Bard and L. R. Faulkner, Electrochemical methods: Fundamentals and applications, John Wiley \& Sons, New York, USA, 2nd edn, 2001.

56 Y. Xu, Z. Lin, X. Huang, Y. Liu, Y. Huang and X. Duan, ACS Nano, 2013, 7, 4042.

57 H. A. Andreas, J. Electrochem. Soc., 2015, 162, A5047.

58 T. Christen and M. W. Carlen, J. Power Sources, 2000, 91, 210. 59 J. Zang and X. Li, J. Mater. Chem., 2011, 21, 10965.

60 S. A. Hashmi, ACS Appl. Mater. Interfaces, 2013, 5, 3875.

61 H. Yu, L. Fan, J. Wu, Y. Lin, M. Huang, J. Lin and Z. Lan, RSC Adv., 2012, 2, 6736.

62 S. Roldán, M. Granda, R. Menéndez, R. Santamaría and C. Blanco, J. Phys. Chem. C, 2011, 115, 17606.

63 S. T. Senthilkumar, R. K. Selvan, Y. S. Lee and J. S. Melo, J. Mater. Chem. A, 2013, 1, 1086.

64 J. Zhong, L.-Q. Fan, X. Wu, J.-H. Wu, G.-J. Liu, J.-M. Lin, M.-L. Huang and Y.-L. Wei, Electrochim. Acta, 2015, 166, 150. 65 G. Ma, M. Dong, K. Sun, E. Feng, H. Peng and Z. Lei, J. Mater. Chem. A, 2015, 3, 4035.

66 D. Li and R. B. Kaner, Chem. Commun., 2005, 3286.

67 J. Huang and R. B. Kaner, Angew. Chem., Int. Ed., 2004, 43, 5817; Angew. Chem., 2004, 116, 5941.

68 J. Huang and R. B. Kaner, Chem. Commun., 2006, 367. 